\title{
Dynamic reduction strategies to extend modal analysis approach at higher frequencies
}

\author{
Pietro Salvini*, Francesco Vivio \\ Department of Mechanical Engineering, University of Rome "Tor Vergata", Via del Politecnico 1, 00133 Rome, Italy
}

Received 5 July 2006; received in revised form 7 June 2007; accepted 7 June 2007

Available online 26 July 2007

\begin{abstract}
Modal analysis on huge finite element models requires a numerical simplification in terms of total number of degrees of freedom (d.o.f.'s). The above aim is generally achieved by choosing some master d.o.f.'s and condensing the structure matrices on those d.o.f.'s. Static condensation (i.e. stiffness matrix reduction) is theoretically an errorless operation; on the other hand, mass condensation can only be approximate in dynamic applications. In order to accomplish matrix condensation, the technique mainly used is the so-called Guyan reduction. The present paper outlines the limitations of the technique, introducing some significant improvements. These are related to the inertia conservation properties of the reduced mass matrix and the condensed mass matrix assembly by means of fictitious and appropriate stiffness connections that are different from those obtained by the stiffness model. The effectiveness of the modified approach is demonstrated with respect to the modal analysis results obtained by Guyan approach, through three different test cases.
\end{abstract}

(C) 2007 Elsevier B.V. All rights reserved.

Keywords: Finite element; Modal analysis; Mass matrix; Guyan reduction

\section{Introduction}

Eigenanalysis is often a starting point in evaluating the behaviour of structures subjected to dynamic loads. The resulting eigenvalues-connected to free vibration frequencies of structures-and eigenvectors, providing mode shapes coupled to each mode of free vibration, are used as primary information to reduce the numerical complexity in the study of dynamic systems.

In the modal analysis of structures damping is rarely considered as it causes a doubling of the degrees of freedom (d.o.f.'s); nonetheless, finite element analysis often involves tens or hundreds thousands of d.o.f.'s. Therefore, as the computational effort required to perform a full modal analysis is generally overwhelming, the reduction of the effective number of d.o.f.'s is mandatory.

Some of the existing methods exploit the component mode synthesis technique [1,2]; these approaches considerably

\footnotetext{
* Corresponding author. Tel.: +3906 72597140; fax: +39062021351.

E-mail addresses: salvini@uniroma2.it (P. Salvini), vivio@uniroma2.it (F. Vivio)
}

simplify the analysis. However, they do not provide full reduced matrices for representing distributed inertia and stiffness properties of the original system. Several algorithms have also been developed to identify new reduced matrices (stiffness and mass matrices) that embody the system characteristics in the specified approximation $[3,4]$.

The basic idea is to focus only on a subset of the total number of d.o.f.'s, the so called "master d.o.f.'s", and try to condense on them all elastic and inertia loads acting on the structure. The vanishing d.o.f.'s are called "slaves d.o.f.'s", since the method forces their values to depend only on master d.o.f.'s.

The most widespread procedure to condense stiffness and mass matrices is the Guyan reduction [3]. It is generally known as static condensation: although it is offered as default in almost all finite element commercial codes, its precision is guaranteed only when excitation frequency is close to zero (i.e. static analysis). Stiffness matrix reduction is exact while mass reduction requires neglecting all inertia loads with respect to elastic loads.

It has been demonstrated that the static procedure allows extending the evaluation of mode shapes within the limit of $0.3 f_{\mathrm{s}}$, being $f_{\mathrm{s}}$ the smallest natural frequency of the structure 
when all master d.o.f.'s are restrained [5]. Therefore, if masters are properly chosen, the reliability of lower mode shapes is assumed. On the contrary, higher modes are completely missing.

Commercial F.E. codes generally adopt very simple approaches in the selection of master d.o.f.'s even if better criteria can be found in literature, (e.g. [6]).

Besides Guyan procedure, several dynamic condensations have been also developed, originating from the work of Leung [7]. All account for inertia effects that modify the dynamic stiffness matrix resulting in a dependency on excitation frequency. The above link is non trivial; therefore, many methods, e.g. the improved reduced system (IRS) proposed by O'Challagan [8], expand in Taylor series the frequency-dependent terms. Expansion is generally truncated to quadratic terms in the effort of extending the reduction to higher frequencies.

It should be noted that Guyan reduction is centred on zero excitation frequency, but it can be easily shifted to higher frequencies by computing the exact stiffness dynamic matrix case by case. The result is a condensed system whose precision is guaranteed only in the surroundings of the new shifted centred frequency.

The method translated into an iterative variant by Friswell et al. $[9,10]$, also identifying its convergence rate, that depends tightly on master d.o.f. selection. Other iterative procedures have been proposed by Kim and Kang [11] and, more recently, by Lin et al. [4] reaching an iterative solution that avoids inertia term truncation.

\section{Basic formulation}

Free vibrations of undamped structures is referenced by the classic equation

$\left(\mathbf{K}-\lambda^{(i)} \mathbf{M}\right) \mathbf{u}^{(i)}=0 ; \quad i=1, \ldots, n$

where $\mathbf{M}$ and $\mathbf{K}$ are $n \times n$ symmetric mass and stiffness matrices, respectively, $\lambda^{(i)}$ is the generic eigenvalue and $\mathbf{u}^{(i)}$ is the corresponding eigenvector.

The first step reduces the system into a simpler one by splitting the d.o.f.'s into masters and slaves, so that $m+s=n$. Matrices can be partitioned accordingly; by omitting apex (i) on eigenvalues for sake of clarity, it results:

$$
\left(\left[\begin{array}{cc}
\mathbf{K}_{\mathbf{m m}} & \mathbf{K}_{\mathbf{m s}} \\
\mathbf{K}_{\mathbf{s m}} & \mathbf{K}_{\mathbf{s s}}
\end{array}\right]-\lambda\left[\begin{array}{cc}
\mathbf{M}_{\mathbf{m m}} & \mathbf{M}_{\mathbf{m s}} \\
\mathbf{M}_{\mathbf{s m}} & \mathbf{M}_{\mathbf{s s}}
\end{array}\right]\right)\left\{\begin{array}{l}
\mathbf{u}_{\mathbf{m}} \\
\mathbf{u}_{\mathbf{s}}
\end{array}\right\}=0
$$

By placing slave and master d.o.f.'s in the left- and right-hand side, respectively, the second set of Eq. (2) yields:

$\left(\mathbf{K}_{\mathrm{ss}}-\lambda \mathbf{M}_{\mathrm{ss}}\right) \mathbf{u}_{\mathrm{s}}=-\left(\mathbf{K}_{\mathrm{sm}}-\lambda \mathbf{M}_{\mathrm{sm}}\right) \mathbf{u}_{\mathbf{m}}$.

It is now possible to solve $\mathbf{u}_{s}$ by premultiplying Eq. (3) for an inverse matrix:

$\mathbf{u}_{s}=-\left[\left(\mathbf{K}_{\mathrm{ss}}-\lambda \mathbf{M}_{\mathbf{s s}}\right)^{-1}\left(\mathbf{K}_{\mathbf{s m}}-\lambda \mathbf{M}_{\mathbf{s m}}\right)\right] \mathbf{u}_{\mathbf{m}}=\mathbf{t}_{\mathbf{s m}}(\lambda) \mathbf{u}_{\mathbf{m}}$.

Therefore, $\mathbf{t}_{\mathbf{s m}}$ turns out to be a transformation matrix, dependent on frequency by means of $\lambda$. According to Eq. (4), transformation of eigenvectors from the full set of d.o.f.'s to the master set is

$\mathbf{u}=\left\{\begin{array}{c}\mathbf{u}_{\mathbf{m}} \\ \mathbf{u}_{\mathbf{s}}\end{array}\right\}=\left[\begin{array}{c}\mathbf{I}_{\mathbf{m m}} \\ \mathbf{t}_{\mathbf{s m}}(\lambda)\end{array}\right] \mathbf{u}_{\mathbf{m}}=\mathbf{T}_{\mathbf{n m}}(\lambda) \mathbf{u}_{\mathbf{m}}$

Eq. (5) can be used to substitute eigenvectors in Eq. (1), obtaining:

$\left[\mathbf{K T}_{\mathbf{n m}}(\lambda)-\lambda \mathbf{M T} \mathbf{T}_{\mathbf{n m}}(\lambda)\right] \mathbf{u}_{\mathbf{m}}=\mathbf{0}$.

Premultiplying by $\mathbf{T}_{\mathbf{n m}}^{\mathrm{T}}$, symmetric stiffness and mass reduced matrices are obtained

$\left[\mathbf{T}_{\mathbf{n m}}^{\mathrm{T}}(\lambda) \mathbf{K} \mathbf{T}_{\mathbf{n m}}(\lambda)-\lambda \mathbf{T}_{\mathbf{n m}}^{\mathrm{T}}(\lambda) \mathbf{M} \mathbf{T}_{\mathbf{n m}}(\lambda)\right] \mathbf{u}_{\mathbf{m}}=0 ;$

$\mathbf{K}_{\mathbf{R}}(\lambda)=\mathbf{T}_{\mathbf{n m}}^{\mathrm{T}}(\lambda) \mathbf{K T}_{\mathbf{n m}}(\lambda) ; \quad \mathbf{M}_{\mathbf{R}}(\lambda)=\mathbf{T}_{\mathbf{n m}}^{\mathrm{T}}(\lambda) \mathbf{M T}_{\mathbf{n m}}(\lambda)$.

This reduction is exact only for a given eigenvalue. The application on different eigenfrequencies thus implies variability of stiffness and mass matrix elements.

An equivalent reduction holds if the system is harmonically excited only on master d.o.f.'s:

$\left\{\left[\begin{array}{cc}\mathbf{K}_{\mathrm{mm}} & \mathbf{K}_{\mathrm{ms}} \\ \mathbf{K}_{\mathrm{sm}} & \mathbf{K}_{\mathrm{ss}}\end{array}\right]-\omega^{2}\left[\begin{array}{cc}\mathbf{M}_{\mathrm{mm}} & \mathbf{M}_{\mathbf{m s}} \\ \mathbf{M}_{\mathbf{s m}} & \mathbf{M}_{\mathrm{ss}}\end{array}\right]\right\}\left\{\begin{array}{c}\mathbf{x}_{\mathbf{m}} \\ \mathbf{x}_{\mathrm{s}}\end{array}\right\}=\left\{\begin{array}{c}\mathbf{f}_{\mathbf{m}} \\ \mathbf{0}_{\mathrm{s}}\end{array}\right\}$.

Reduction is achieved with manipulations that are similar to those previously applied, thus giving generality to the identified mass and stiffness reduction matrices.

$$
\begin{aligned}
& {\left[\mathbf{T}_{\mathbf{n m}}^{\mathrm{T}}(\omega) \mathbf{K} \mathbf{T}_{\mathbf{n m}}(\omega)-\omega^{2} \mathbf{T}_{\mathbf{n m}}^{\mathrm{T}}(\omega) \mathbf{M} \mathbf{T}_{\mathbf{n m}}(\omega)\right] \mathbf{x}_{\mathbf{m}}} \\
& \quad=\mathbf{T}_{\mathbf{n m}}^{\mathrm{T}}(\omega) \mathbf{f}_{\mathbf{m}}(\omega) ;
\end{aligned}
$$

$\mathbf{K}_{\mathbf{R}}(\omega)=\mathbf{T}_{\mathbf{n m}}^{\mathrm{T}}(\omega) \mathbf{K} \mathbf{T}_{\mathbf{n m}}(\omega) ; \quad \mathbf{M}_{\mathbf{R}}(\omega)=\mathbf{T}_{\mathbf{n m}}^{\mathrm{T}}(\omega) \mathbf{M} \mathbf{T}_{\mathbf{n m}}(\omega)$.

The computational cost of above reductions is embedded into Eq. (4): the inversion of the slave dynamic stiffness matrix is required at any circular frequency.

Guyan approximation neglects both inertia terms of Eq. (4); the same result is achieved when Eq. (4) is specialised for $\lambda=0$. As a consequence, the transformation matrix $\mathbf{T}_{\mathbf{n m}}^{\mathbf{G}}$, simplifies into:

$\mathbf{T}_{\mathbf{n m}}^{\mathbf{G}}=\left[\begin{array}{c}\mathbf{I}_{\mathrm{mm}} \\ \mathbf{t}_{\mathrm{sm}}^{\mathbf{G}}\end{array}\right]=\left[\begin{array}{c}\mathbf{I}_{\mathrm{mm}} \\ -\mathbf{K}_{\mathrm{ss}}^{-1} \mathbf{K}_{\mathrm{sm}}\end{array}\right]$.

After expansion of either Eq. (8) or (11), two definitions result: the first is lighter, taking advantage of several simplifications, the other one turns out to be heavier

$$
\begin{aligned}
\mathbf{K}_{\mathbf{R}}= & \left(\mathbf{K}_{\mathbf{m m}}-\mathbf{K}_{\mathrm{ms}} \mathbf{K}_{\mathrm{ss}}^{-1} \mathbf{K}_{\mathrm{sm}}\right) ; \\
\mathbf{M}_{\mathbf{R}}= & \mathbf{T}_{\mathbf{n m}}^{\mathrm{T}} \mathbf{M} \mathbf{T}_{\mathbf{n m}} \\
= & \left(\mathbf{M}_{\mathrm{mm}}-\mathbf{M}_{\mathrm{ms}} \mathbf{K}_{\mathrm{ss}}^{-1} \mathbf{K}_{\mathrm{sm}}-\mathbf{K}_{\mathrm{ms}} \mathbf{K}_{\mathrm{ss}}^{-1} \mathbf{M}_{\mathrm{sm}}\right. \\
& \left.+\mathbf{K}_{\mathrm{ms}} \mathbf{K}_{\mathrm{ss}}^{-1} \mathbf{M}_{\mathrm{ss}} \mathbf{K}_{\mathrm{ss}}^{-1} \mathbf{K}_{\mathrm{sm}}\right) .
\end{aligned}
$$


It appears evident that transformation matrix (12) accounts only for the stiffness characteristics of the slave d.o.f.'s and the connections between master and slaves d.o.f.'s.

\section{A new approach to reduce mass matrix}

As described in the previous section, Guyan approach computes the reduced mass matrix taking into account the elastic characteristics of the system that result from the stiffness matrix.

At this stage a question arises: why should the inertia properties of a mechanical system, depending on mass distribution morphology, be affected only by stiffness characteristics of slave nodes? From an other point of view, could two mechanical systems, having an identical mass distribution but dissimilar elastic properties, reasonably present different reduced mass matrices?

A consequence of the above queries is that, in principle, any stiffness matrix can be used when applying Eq. (14). This conclusion involves a new reduced formulation based on a general stiffness matrix $\mathbf{K}^{(\mathbf{G})}$ (non necessarily coincident with the previous $\mathbf{K}$ corresponding to the actual mechanical system):

$$
\begin{aligned}
\mathbf{M}_{\mathbf{R}}= & \mathbf{M}_{\mathbf{m m}}-\mathbf{M}_{\mathbf{m s}}\left(\mathbf{K}_{\mathrm{ss}}^{(\mathbf{G})}\right)^{-\mathbf{1}} \mathbf{K}_{\mathrm{sm}}^{(\mathbf{G})}-\mathbf{K}_{\mathrm{ms}}^{(\mathbf{G})}\left(\mathbf{K}_{\mathrm{ss}}^{(\mathbf{G})}\right)^{-\mathbf{1}} \mathbf{M}_{\mathbf{s m}} \\
& +\mathbf{K}_{\mathbf{m s}}^{(\mathbf{G})}\left(\mathbf{K}_{\mathrm{ss}}^{(\mathbf{G})}\right)^{-\mathbf{1}} \mathbf{M}_{\mathbf{s s}}\left(\mathbf{K}_{\mathrm{ss}}^{(\mathbf{G})}\right)^{-\mathbf{1}} \mathbf{K}_{\mathrm{sm}}^{(\mathbf{G})} .
\end{aligned}
$$

The open point is whether the stiffness matrix of the system $\mathbf{K}$ is the finest choice to obtain a proper reduced inertia matrix. As an example, the increase of system elastic connections (i.e. the removal of some zero terms in the stiffness matrix $\mathbf{K}$ ) can share out the inertia effects more suitably; a better approximation at higher frequencies is hence expected.

Nonetheless, stiffness and inertia properties are both considered in choosing master d.o.f.'s, when a particular ratio of reduction is requested. The simplest way is to order the d.o.f.'s according to decreasing values of the ratio $\sqrt{k_{i i} / m_{i i}}$ on diagonal terms. If the frontal solver approach is used, however, the final selection of master d.o.f.'s is influenced by internal element numbering [12]; therefore, outcomes may result as the consequence of different element sorting. To overcome this ambiguity source, in all examples hereinafter discussed, the solution was carried out by managing all d.o.f.'s together, thus avoiding the use of frontal solvers.

The most general choice of $\mathbf{K}^{(\mathbf{G})}$ is a large band stiffness matrix, generated by connecting all d.o.f.'s each other through a given $k$ value. The stiffness matrix results assembled by identical elements $(-k)$ off of the principal diagonal and identical elements $(n-1) k$ in the principal diagonal. It is interesting to show that, if one alters the $\mathbf{K}^{(\mathbf{G})}$ matrix, e.g. considering random values for each $k$ but keeping main diagonal terms as the sum of the corresponding row, $\mathbf{M}_{\mathbf{R}}$ results only slightly modified as the consequence of numerical approximation. The interpretation of the above result is that $\mathbf{M}_{\mathbf{R}}$ is influenced by $\mathbf{K}^{(\mathbf{G})}$ structure (i.e. $\mathbf{K}_{\mathbf{s s}}^{(\mathbf{G})}$ and $\mathbf{K}_{\mathbf{m s}}^{(\mathbf{G})}=\mathbf{K}_{\mathbf{s m}}^{(\mathbf{G})}$ ) rather than by its numerical values. On the contrary, various reduced mass matrices result when a different weight is given to each row (and according column) of matrix $\mathbf{K}^{(\mathbf{G})}$.

\subsection{Preservation of global mass}

A considerable improvement concerns the enforcement of model mass conservation after condensation. As it is here demonstrated, Guyan mass reduction does preserve model mass only under some circumstances.

The total mass of a discretized system can be computed by projecting mass matrix towards a generic rigid mode $\mathbf{r}$ :

$m_{\text {tot }}=\mathbf{r}^{\mathrm{t}} \mathbf{M r}$.

Calling $\mathbf{r}_{\mathbf{m}}$ the rigid mode that concerns only the master d.o.f.'s, reduced model mass computation gives:

$m_{R, \text { tot }}=\mathbf{r}_{\mathbf{m}}^{\mathbf{t}} \mathbf{M}_{\mathbf{R}} \mathbf{r}_{\mathbf{m}}$.

Moreover, in unrestrained structures, the rigid modes satisfy

$\mathbf{K r}=\mathbf{0}$,

which can be rewritten according to master/slave partitioning:

$\left[\begin{array}{ll}\mathbf{K}_{\mathbf{m m}} & \mathbf{K}_{\mathbf{m s}} \\ \mathbf{K}_{\mathrm{sm}} & \mathbf{K}_{\mathrm{ss}}\end{array}\right]\left\{\begin{array}{c}\mathbf{r}_{\mathbf{m}} \\ \mathbf{r}_{\mathbf{s}}\end{array}\right\}=\mathbf{0} ;$

hence, referring to the second line of above equation:

$\mathbf{r}_{\mathbf{s}}=-\mathbf{K}_{\mathrm{ss}}^{-1} \mathbf{K}_{\mathrm{sm}} \mathbf{r}_{\mathbf{m}}$

Coming back to the mass computed by means of the mass matrix reduced by Guyan (Eq. (14)):

$m_{\mathrm{red}, \mathrm{tot}}=\mathbf{r}_{\mathbf{m}}^{\mathbf{t}}\left[\mathbf{I}_{\mathbf{m m}} \quad-\mathbf{K}_{\mathbf{m s}} \mathbf{K}_{\mathbf{s s}}^{-\mathbf{1}}\right] \mathbf{M}\left[\begin{array}{c}\mathbf{I}_{\mathbf{m m}} \\ -\mathbf{K}_{\mathbf{s s}}^{-\mathbf{1}} \mathbf{K}_{\mathbf{s m}}\end{array}\right] \mathbf{r}_{\mathbf{m}}$.

Eq. (20), valid for an unrestrained structure, can be used to simplify previous equation:

$m_{\text {red,tot }}=\left\{\begin{array}{ll}\mathbf{r}_{\mathbf{m}}^{\mathbf{t}} & \mathbf{r}_{\mathbf{s}}^{\mathbf{t}}\end{array}\right\} \mathbf{M}\left(\begin{array}{c}\mathbf{r}_{\mathbf{m}}^{\mathbf{t}} \\ \mathbf{r}_{\mathbf{s}}^{\mathbf{t}}\end{array}\right)$.

Then, in case of unrestrained structures, being $\mathbf{r}=\left\{\begin{array}{ll}\mathbf{r}_{\mathbf{m}}^{\mathbf{t}} & \mathbf{r}_{\mathbf{s}}^{\mathbf{t}}\end{array}\right.$, one obtains the expected result $m_{\text {red,tot }}=m_{\text {tot }}$.

On the contrary, when some d.o.f.'s constraints are experienced by the structure, Eq. (18) is no more valid, therefore it is impossible to obtain Eq. (22) starting from Eq. (21) and using Eq. (20); therefore $m_{\text {red,tot }}$ is no more equal to $m_{\text {tot }}$.

With the aim to evaluate the mass that is eventually lost during reduction, it is possible to consider the following approach. The d.o.f.'s that are to be constrained are identified by subscript $b$ (blocked) and the free d.o.f.'s by subscript $f$; according to this symbol definition, stiffness matrix partition yields:

$\mathbf{K}=\left[\begin{array}{ll}\mathbf{K}_{\mathbf{b b}} & \mathbf{K}_{\mathbf{b f}} \\ \mathbf{K}_{\mathbf{f b}} & \mathbf{K}_{\mathbf{f f}}\end{array}\right]$.

Considering that in a constrained structure $\mathbf{K}_{\mathbf{f f}}$ matrix is the effective matrix to be reduced, master/slave partitioning refers only to it:

$\mathbf{K}_{\mathbf{f f}}=\left[\begin{array}{cc}\mathbf{K}_{\mathrm{mm}} & \mathbf{K}_{\mathrm{ms}} \\ \mathbf{K}_{\mathrm{sm}} & \mathbf{K}_{\mathrm{ss}}\end{array}\right]$. 


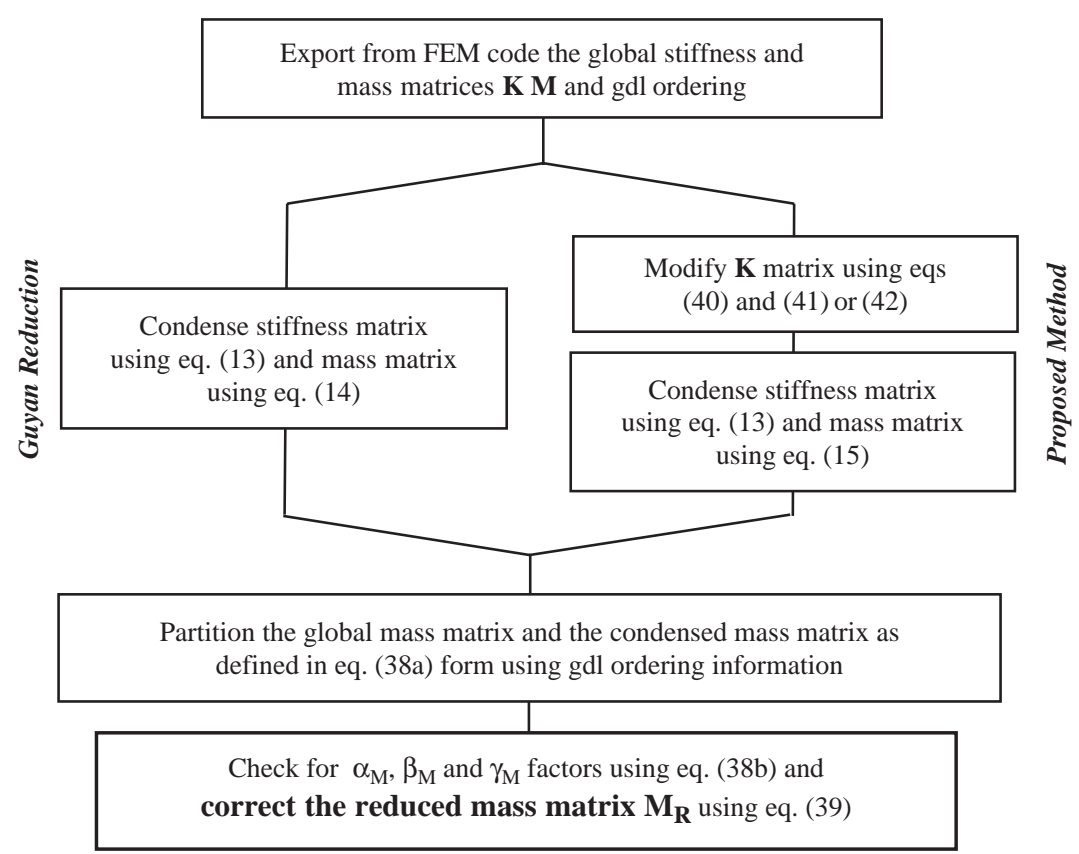

Fig. 1. Logical flow for the use of the mass correction and the application of the modified $\mathbf{K}^{(\mathbf{G})}$.

It is interesting to partition $\mathbf{K}_{\mathbf{b f}}$, accounting of free d.o.f.'s adjacent to the blocked ones, into two parts, according to master and slave free d.o.f.'s:

$\mathbf{K}_{\mathbf{b f}}=\left[\begin{array}{ll}\mathbf{K}_{\mathbf{b f}} & \mathbf{K}_{\mathbf{b f}}\end{array}\right]=\mathbf{K}_{\mathbf{f b}}^{\mathbf{T}}$.

Then, the global unrestrained stiffness matrix results partitioned in the following way:

$\mathbf{K}=\left[\begin{array}{ccc}\mathbf{K}_{\mathbf{b b}} & \mathbf{K}_{\mathbf{b f}} & \mathbf{K}_{\mathbf{b f}_{\mathrm{s}}} \\ \mathbf{K}_{\mathbf{f}_{\mathbf{m}} \mathbf{b}} & \mathbf{K}_{\mathbf{m m}} & \mathbf{K}_{\mathbf{m s}} \\ \mathbf{K}_{\mathbf{f}_{\mathrm{s}} \mathbf{b}} & \mathbf{K}_{\mathbf{s m}} & \mathbf{K}_{\mathbf{s s}}\end{array}\right]$.

The same partition can be applied to virtual global rigid mode $\mathbf{r}$ :

$\mathbf{r}=\left\{\begin{array}{l}\mathbf{r}_{\mathbf{b}} \\ \mathbf{r}_{\mathbf{f}}\end{array}\right\}=\left\{\begin{array}{l}\mathbf{r}_{\mathbf{b}} \\ \mathbf{r}_{\mathbf{m}} \\ \mathbf{r}_{\mathbf{s}}\end{array}\right\}$.

Now, the condition expressed by Eq. (18), can be rewritten:

$\mathbf{K r}=\mathbf{0}=\left[\begin{array}{ll}\mathbf{K}_{\mathbf{b b}} & \mathbf{K}_{\mathbf{b f}} \\ \mathbf{K}_{\mathbf{f b}} & \mathbf{K}_{\mathbf{f f}}\end{array}\right]\left\{\begin{array}{l}\mathbf{r}_{\mathbf{b}} \\ \mathbf{r}_{\mathbf{f}}\end{array}\right\} ;$

looking at the second line of previous equation:

$\mathbf{K}_{\mathbf{f f}} \mathbf{r}_{\mathbf{f}}=-\mathbf{K}_{\mathbf{f b}} \mathbf{r}_{\mathbf{b}}$.

Considering Eqs. (24) and (25), and accounting of $\mathbf{r}_{\mathbf{f}}=\left\{\begin{array}{ll}\mathbf{r}_{\mathbf{m}}^{\mathbf{t}} & \mathbf{r}_{\mathbf{s}}^{\mathbf{t}}\end{array}\right\}$, above equation is:

$\left[\begin{array}{cc}\mathbf{K}_{\mathbf{m m}} & \mathbf{K}_{\mathbf{m s}} \\ \mathbf{K}_{\mathrm{sm}} & \mathbf{K}_{\mathrm{ss}}\end{array}\right]\left\{\begin{array}{c}\mathbf{r}_{\mathrm{m}} \\ \mathbf{r}_{\mathbf{s}}\end{array}\right\}=-\left[\begin{array}{cc}\mathbf{K}_{\mathbf{f}_{\mathrm{m}} \mathbf{b}} & \mathbf{r}_{\mathbf{b}} \\ \mathbf{K}_{\mathbf{f}_{\mathrm{s}} \mathbf{b}} & \mathbf{r}_{\mathbf{b}}\end{array}\right]$.
Focusing attention at the second line, one obtains:

$\mathbf{K}_{\mathrm{sm}} \mathbf{r}_{\mathbf{m}}+\mathbf{K}_{\mathrm{ss}} \mathbf{r}_{\mathrm{s}}=-\mathbf{K}_{\mathbf{f}_{\mathrm{s}} \mathbf{b}} \mathbf{r}_{\mathbf{b}}$

$\mathbf{r}_{\mathrm{s}}=-\mathbf{K}_{\mathrm{ss}}^{-1} \mathbf{K}_{\mathrm{sm}} \mathbf{r}_{\mathrm{m}}-\mathbf{K}_{\mathrm{ss}}^{-1} \mathbf{K}_{\mathbf{f}_{\mathbf{s}}} \mathbf{b} \mathbf{r}_{\mathbf{b}}$.

Remembering the total mass given after Guyan reduction (Eq. (14)) and exploiting Eq. (32):

$$
\begin{aligned}
m_{\text {red,tot }} & =\mathbf{r}_{\mathbf{m}}^{\mathbf{T}}\left[\mathbf{I}_{\mathbf{m m}}-\mathbf{K}_{\mathbf{m s}} \mathbf{K}_{\mathrm{ss}}^{-1}\right] \mathbf{M}_{\mathbf{f f}}\left[\begin{array}{c}
\mathbf{I}_{\mathbf{m m}} \\
-\mathbf{K}_{\mathrm{ss}}^{-1} \mathbf{K}_{\mathbf{s m}}
\end{array}\right] \mathbf{r}_{\mathbf{m}} \\
& =\left[\begin{array}{ll}
\mathbf{r}_{\mathbf{m}}^{\mathbf{T}} & -\mathbf{r}_{\mathbf{m}}^{\mathbf{T}} \mathbf{K}_{\mathbf{m s}} \mathbf{K}_{\mathrm{ss}}^{-1}
\end{array}\right] \mathbf{M}_{\mathbf{f f}}\left[\begin{array}{c}
\mathbf{r}_{\mathbf{m}} \\
-\mathbf{K}_{\mathrm{ss}}^{-1} \mathbf{K}_{\mathrm{sm}} \mathbf{r}_{\mathbf{m}}
\end{array}\right] \\
& =\left[\begin{array}{ll}
\mathbf{r}_{\mathbf{m}}^{\mathbf{T}} & \mathbf{r}_{\mathbf{s}}^{\mathbf{T}}+\mathbf{r}_{\mathbf{b}}^{\mathbf{T}} \mathbf{K}_{\mathbf{b f}} \mathbf{K}_{\mathrm{ss}}^{-1}
\end{array}\right] \mathbf{M}_{\mathbf{f f}}\left[\begin{array}{c}
\mathbf{r}_{\mathbf{m}} \\
\mathbf{r}_{\mathbf{s}}+\mathbf{K}_{\mathrm{ss}}^{-1} \mathbf{K}_{\mathbf{f}_{\mathbf{s}} \mathbf{b}} \mathbf{r}_{\mathbf{b}}
\end{array}\right],
\end{aligned}
$$

and given that

$m_{\text {tot }}=\mathbf{r}_{\mathbf{f}}^{\mathbf{T}} \mathbf{M}_{\mathbf{f f}} \mathbf{r}_{\mathbf{f}}=\left[\begin{array}{ll}\mathbf{r}_{\mathbf{m}}^{\mathbf{T}} & \mathbf{r}_{\mathbf{s}}^{\mathbf{T}}\end{array}\right] \mathbf{M}_{\mathbf{f f}}\left\{\begin{array}{c}\mathbf{r}_{\mathbf{m}} \\ \mathbf{r}_{\mathbf{s}}\end{array}\right\}$,

it is possible to deduce, by comparison of Eqs. (33) and (34), that only if $\mathbf{K}_{\mathbf{f}_{\mathbf{s}} \mathbf{b}}=\mathbf{0}$ it results that $m_{\text {tot }}=m_{\text {red,tot }}$.

The resulting outcome is that global mass is conserved only if there are no slave d.o.f.'s connected to any constrained d.o.f. Therefore, in a substructuring analysis, an attention should be paid when master d.o.f.'s have stiffness connections with restrained d.o.f.'s. Of course, this restriction has an effect on Mass computation, while the reduced stiffness matrix keeps exact in any case. 

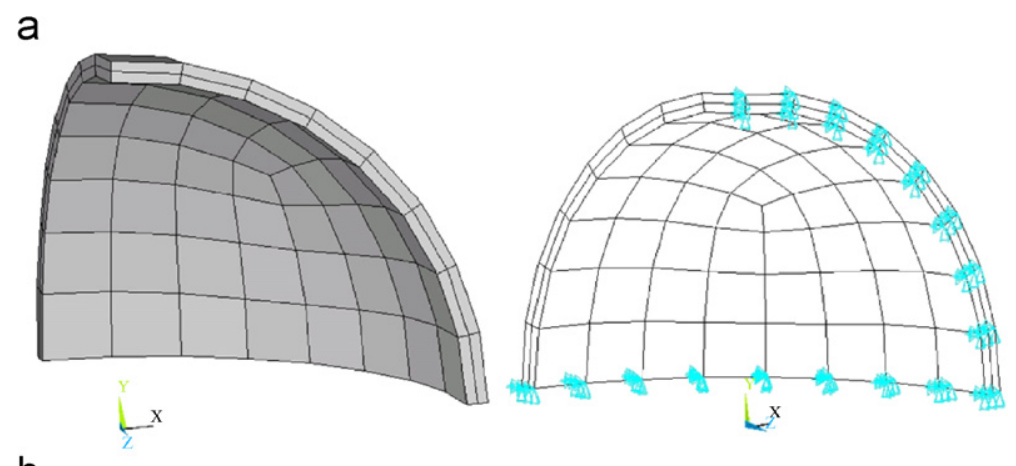

b
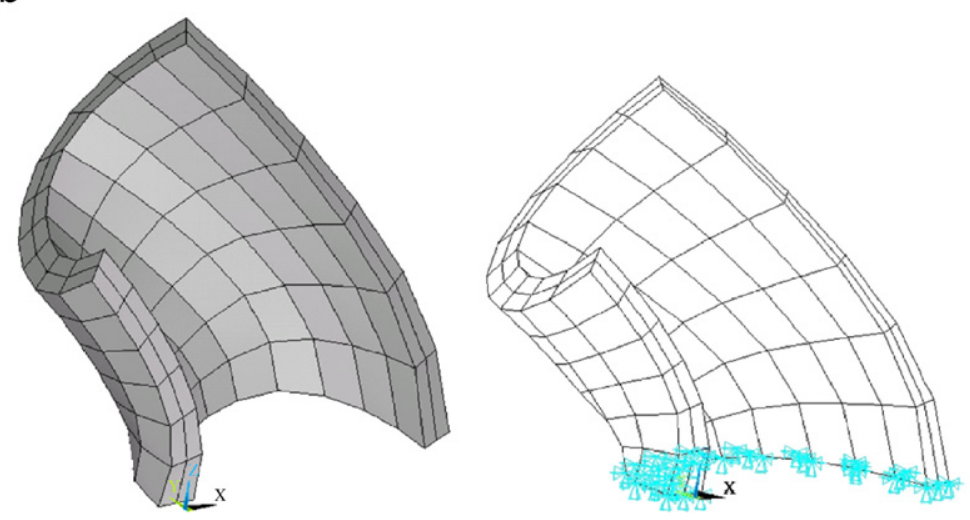

C
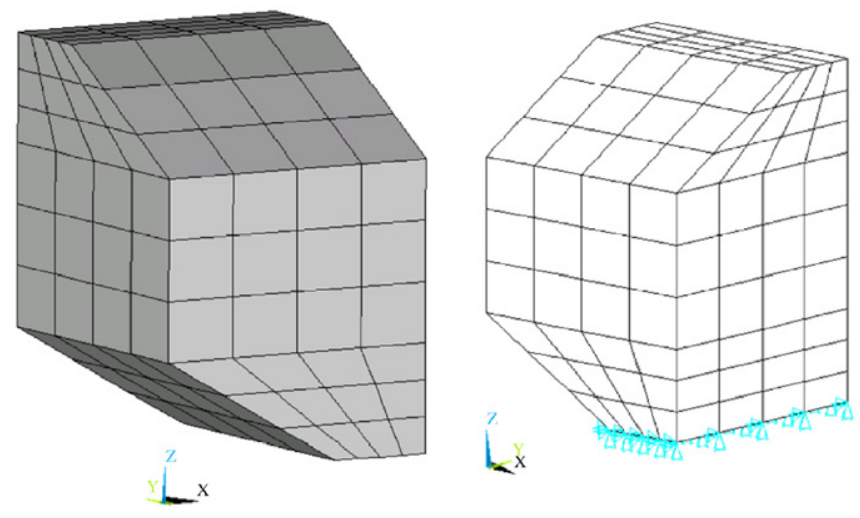

Fig. 2. Models used in condensation procedure: (a) Model 1 (396 d.o.f.'s); (b) Model 2 (594 d.o.f.'s); and (c) Model 3 (675 d.o.f.'s).

Calling now $\mathbf{r}_{\mathbf{s}}^{*}=\mathbf{r}_{\mathbf{s}}+\mathbf{K}_{\mathrm{ss}}^{-\mathbf{1}} \mathbf{K}_{\mathbf{f}_{\mathbf{s}} \mathbf{b}} \mathbf{r}_{\mathbf{b}}$, the mass of the reduced structure is

$$
\begin{aligned}
m_{\text {red,tot }}= & {\left[\begin{array}{ll}
\mathbf{r}_{\mathbf{m}}^{\mathbf{T}} & \mathbf{r}_{\mathbf{s}}^{* \mathbf{T}}
\end{array}\right] \mathbf{M}_{\mathbf{f f}}\left[\begin{array}{c}
\mathbf{r}_{\mathbf{m}} \\
\mathbf{r}_{\mathbf{s}}^{*}
\end{array}\right]=\left[\begin{array}{ll}
\mathbf{r}_{\mathbf{m}}^{\mathbf{T}} & \mathbf{r}_{\mathbf{s}}^{* \mathbf{T}}
\end{array}\right]\left[\begin{array}{cc}
\mathbf{M}_{\mathbf{m m}} & \mathbf{M}_{\mathbf{m s}} \\
\mathbf{M}_{\mathbf{s m}} & \mathbf{M}_{\mathbf{s s}}
\end{array}\right]\left[\begin{array}{c}
\mathbf{r}_{\mathbf{m}} \\
\mathbf{r}_{\mathbf{s}}^{*}
\end{array}\right] } \\
= & \mathbf{r}_{\mathbf{m}}^{\mathbf{T}} \mathbf{M}_{\mathbf{m m}} \mathbf{r}_{\mathbf{m}}+\mathbf{r}_{\mathbf{m}}^{\mathbf{T}} \mathbf{M}_{\mathbf{m s}} \mathbf{r}_{\mathbf{s}}^{*} \\
& +\mathbf{r}_{\mathbf{s}}^{* \mathbf{T}} \mathbf{M}_{\mathbf{s m}} \mathbf{r}_{\mathbf{m}}+\mathbf{r}_{\mathbf{m}}^{\mathbf{T}} \mathbf{M}_{\mathbf{m s}} \mathbf{r}_{\mathbf{s}}^{*} .
\end{aligned}
$$

Considering that the mass of the whole structure is

$m_{\text {tot }}=\mathbf{r}_{\mathbf{m}}^{\mathbf{T}} \mathbf{M}_{\mathbf{m m}} \mathbf{r}_{\mathbf{m}}+\mathbf{r}_{\mathbf{m}}^{\mathbf{T}} \mathbf{M}_{\mathbf{m s}} \mathbf{r}_{\mathbf{s}}+\mathbf{r}_{\mathbf{s}}^{\mathbf{T}} \mathbf{M}_{\mathbf{s m}} \mathbf{r}_{\mathbf{m}}+\mathbf{r}_{\mathbf{s}}^{\mathbf{T}} \mathbf{M}_{\mathbf{s s}} \mathbf{r}_{\mathbf{s}}$

subtracting Eqs. (36) to (35), after some simple manipulations it is possible to obtain the expression giving the mass lost using Guyan reduction when constraints are introduced in the structure:

$$
\begin{aligned}
m_{\text {red,less }}= & m_{\text {tot }}-m_{\text {red,tot }} \\
= & -\mathbf{r}_{\mathbf{b}}^{\mathbf{T}} \mathbf{K}_{\mathbf{b} \mathbf{f}_{\mathbf{s}}} \mathbf{K}_{\mathbf{s s}}^{-\mathbf{1}} \mathbf{M}_{\mathbf{s m}} \mathbf{r}_{\mathbf{m}}-\mathbf{r}_{\mathbf{b}}^{\mathbf{T}} \mathbf{K}_{\mathbf{b} \mathbf{s}_{\mathbf{s}}} \mathbf{K}_{\mathbf{s s}}^{-\mathbf{1}} \mathbf{M}_{\mathbf{s s}} \mathbf{r}_{\mathbf{s}} \\
& -\left[\mathbf{r}_{\mathbf{m}}^{\mathbf{T}} \mathbf{M}_{\mathbf{m s}}+\mathbf{r}_{\mathbf{s}}^{\mathbf{T}} \mathbf{M}_{\mathbf{s s}}\right. \\
& \left.+\mathbf{r}_{\mathbf{b}}^{\mathbf{T}} \mathbf{K}_{\mathbf{b} \mathbf{f}_{\mathbf{s}}} \mathbf{K}_{\mathbf{s s}}^{-\mathbf{1}} \mathbf{M}_{\mathbf{s s}}\right] \mathbf{K}_{\mathbf{s s}}^{-\mathbf{1}} \mathbf{K}_{\mathbf{f}_{\mathbf{s}} \mathbf{b}} \mathbf{r}_{\mathbf{b}} .
\end{aligned}
$$

This occurrence affects the accuracy of computed eigenvalues, as the following numerical examples show.

Even if previous Eq. (37) gives an exact value of the mass that can be missed during a Guyan reduction, from a computational point of view, a more suitable approach can be adopted.

The computation of global mass in each direction $(x, y, z)$ is straightforward in the modelling of mechanical structures; it can be performed by summing up all mass matrix elements that refer to any direction $(x, y, z)$. 
a

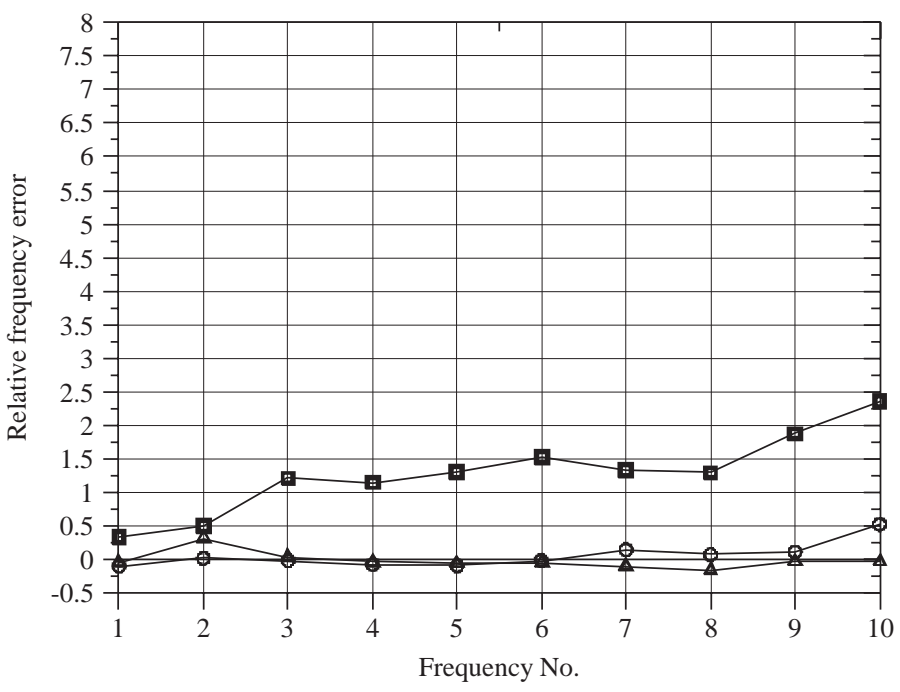

\begin{tabular}{|c|c|c|c|c|c|}
\hline $\begin{array}{l}\text { Freq. } \\
\text { [SI]. }\end{array}$ & $\begin{array}{c}\text { No } \\
\text { condensed }\end{array}$ & $\begin{array}{l}\text { Classic } \\
\text { Guyan }\end{array}$ & $\begin{array}{c}\text { Guyan } \\
\text { corrected }\end{array}$ & $\begin{array}{c}\text { New } \\
\text { method }\end{array}$ & \\
\hline $1^{\mathrm{st}}$ & 581.3 & 767.8 & 520.3 & 551.9 & \multirow{6}{*}{$\frac{2}{2}$} \\
\hline $2^{\text {nd }}$ & 602.7 & 905.0 & 609.6 & 791.8 & \\
\hline $3^{\mathrm{rd}}$ & 925.6 & 2042.0 & 903.7 & 959.5 & \\
\hline $4^{\text {th }}$ & 1168.1 & 2504.3 & 1079.8 & 1119.0 & \\
\hline $5^{\text {th }}$ & 1230.4 & 2834.8 & 1107.0 & 1157.8 & \\
\hline $10^{\text {th }}$ & 1834.2 & 6141.6 & 2787.8 & 1775.9 & \\
\hline $1^{\mathrm{st}}$ & 1323.1 & 1567.5 & 987.2 & 1126.3 & \\
\hline $2^{\text {nd }}$ & 2002.9 & 2613.1 & 1390.2 & 1523.0 & \\
\hline $3^{\text {rd }}$ & 2724.3 & 5530.1 & 2218.7 & 3227.7 & \\
\hline $4^{\text {th }}$ & 3703.1 & 6097.7 & 2918.4 & 4257.1 & \\
\hline $5^{\text {th }}$ & 4222.9 & 10060.8 & 4676.1 & 5024.5 & \\
\hline $10^{\text {th }}$ & 9213.1 & 47257.2 & 35418.7 & 10559.8 & \\
\hline $1^{\mathrm{st}}$ & 169.1 & 184.0 & 136.0 & 129.3 & \\
\hline $2^{\text {nd }}$ & 336.3 & 472.1 & 233.4 & 234.8 & \\
\hline $3^{\text {rd }}$ & 483.4 & 4185.1 & 2564.1 & 536.6 & \\
\hline $4^{\text {th }}$ & 927.7 & 4309.6 & 3136.5 & 538.7 & \\
\hline $5^{\text {th }}$ & 1104.3 & 7990.1 & 4868.2 & 788.5 & \\
\hline $10^{\text {th }}$ & 2564.2 & 15982.3 & 11908.0 & 1330.6 & \\
\hline
\end{tabular}

b

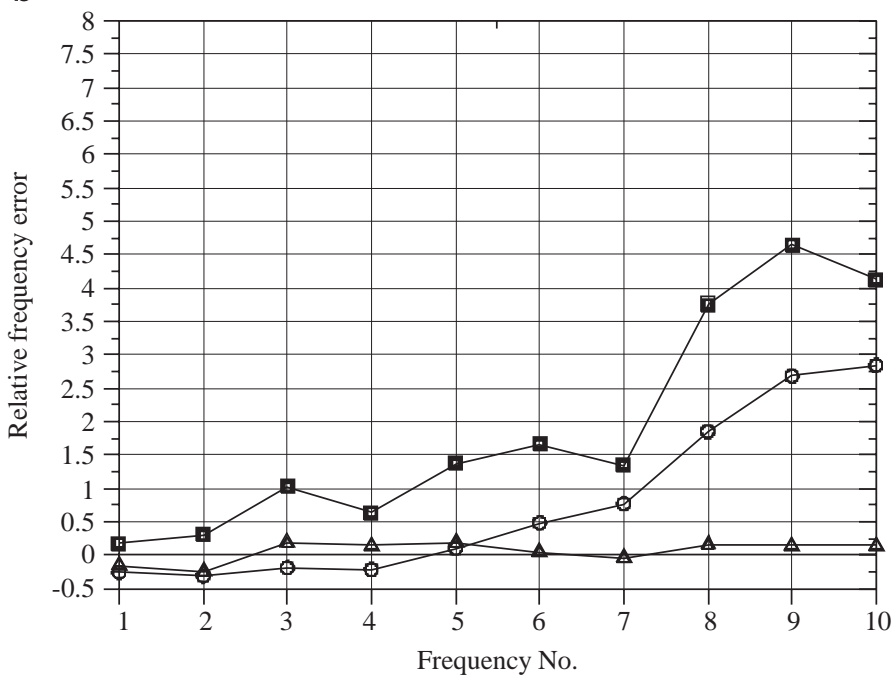

\begin{tabular}{|l|l|l|}
\cline { 2 - 3 } \multicolumn{1}{c|}{} & Guyan corrected & New method \\
\hline $\mathrm{nm}_{\mathrm{x}}=2$ & $\alpha_{\mathrm{M}}=\mathbf{9 . 4 6}$ & $\alpha_{\mathrm{M}}=\mathbf{4 3 4 . 3 7}$ \\
\hline $\mathrm{nm}_{\mathrm{y}}=0$ & $\beta_{\mathrm{M}}=-----$ & $\beta_{\mathrm{M}}=-----$ \\
\hline $\mathrm{nm}_{\mathrm{z}}=8$ & $\gamma_{\mathrm{M}}=\mathbf{1 . 7 5}$ & $\gamma_{\mathrm{M}}=\mathbf{8 1 . 5 8}$ \\
\hline
\end{tabular}

C

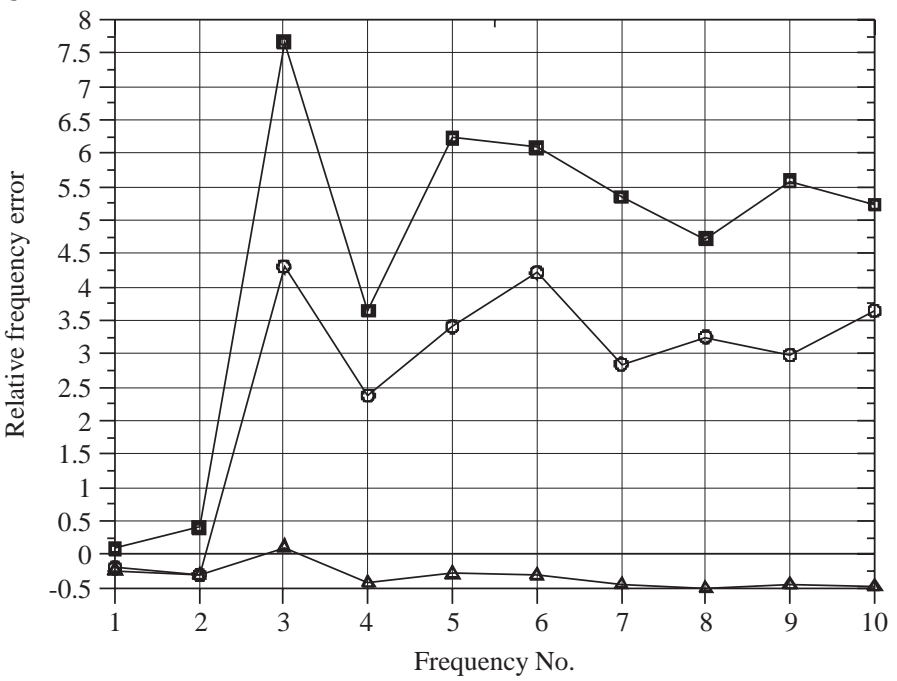

\begin{tabular}{|l|l|l|}
\cline { 2 - 3 } \multicolumn{1}{c|}{} & Guyan corrected & New method \\
\hline $\mathrm{nm}_{\mathrm{x}}=5$ & $\alpha_{\mathrm{M}}=\mathbf{1 . 8 0}$ & $\alpha_{\mathrm{M}}=\mathbf{1 2 1 . 3 4}$ \\
\hline $\mathrm{nm}_{\mathrm{y}}=5$ & $\beta_{\mathrm{M}}=\mathbf{2 . 7 3}$ & $\beta_{\mathrm{M}}=\mathbf{1 2 1 . 3 4}$ \\
\hline $\mathrm{nm}_{\mathrm{z}}=0$ & $\gamma_{\mathrm{M}}=-----$ & $\gamma_{\mathrm{M}}=-----$ \\
\hline
\end{tabular}

Fig. 3. Comparison of eigenfrequency predictions among original Guyan method ( $-\mathbf{E}-$ ), mass corrected Guyan method ( $\longrightarrow \mathbf{O}$ ) and the new developed method (_

Therefore, an improvement of the reduced mass matrix is gained through the introduction of suitable factors that modify the term of the mass matrix, independently in each direction $(x, y, z)$. The above factors update the reduced matrix in order to keep the mass to the value of the original unrestrained structure. This improvement is easily applicable to finite el- ements structures that are modelled through the translational d.o.f.'s elements only: significant and extensive examples are all structures modelled with brick elements. When rotational d.o.f.'s are present, each finite element type present in the model should be examined individually, depending on shape function definition. 


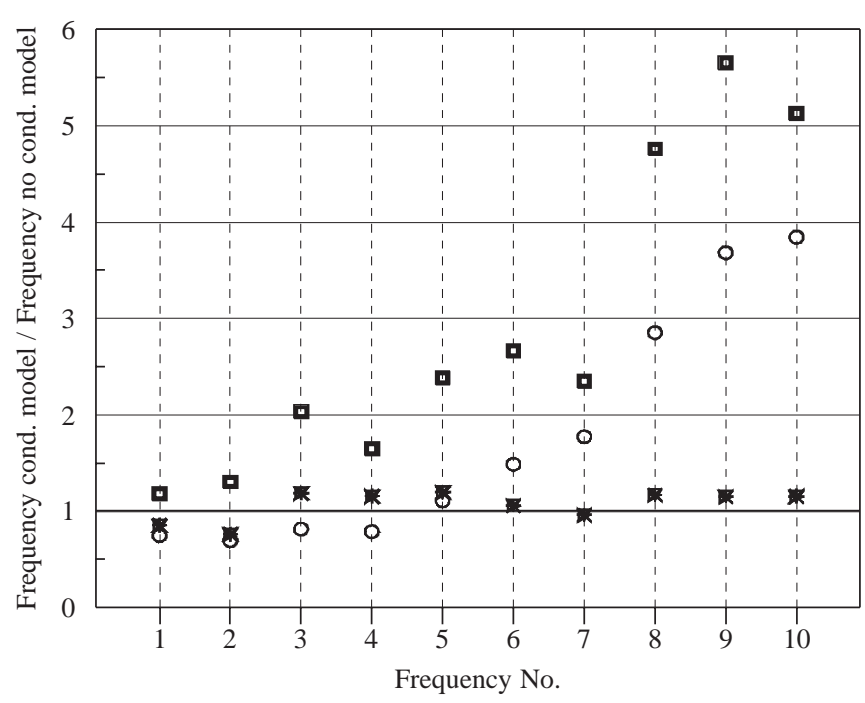

\begin{tabular}{c|l|c|c|c}
\multicolumn{2}{l}{} & $\boldsymbol{\alpha}_{\mathbf{M}}$ & $\boldsymbol{\beta}_{\mathbf{M}}$ & $\boldsymbol{\gamma}_{\mathbf{M}}$ \\
\hline $\mathbf{\square}$ & Guyan & $/$ & $/$ & $/$ \\
\hline$\bigcirc$ & Corrected Guyan & 9.46 & --- & 1.75 \\
\hline$\times$ & $\mathbf{K}^{(\mathbf{G})}$ with $\eta=1 ; \quad \delta=\max \left|k_{i j}\right|$ & 434.37 & --- & 81.58 \\
\hline * & $\mathbf{K}^{(\mathbf{G})}$ with $\eta=-10^{-1} ; \delta=\max \left|k_{i j}\right|$ & 432.61 & --- & 80.37 \\
\hline$\nabla$ & $\mathbf{K}^{(\mathbf{G})}$ with $\eta=0$ & 433.42 & ---- & 80.87 \\
\hline$\triangleright$ & $\mathbf{K}_{\text {TEST }}^{(\mathbf{G})}$ & 434.28 & --- & 81.47
\end{tabular}

Fig. 4. Results obtained using different reduction strategies for model no. 2; 10 master d.o.f.'s $(n m=10), n m_{x}=2, n m_{y}=0, n m_{z}=8$.

The following expressions supply a posteriori mass correction, giving factors $\alpha_{\mathrm{M}}, \beta_{\mathrm{M}}$ and $\gamma_{\mathrm{M}}$ (according to $x, y, z$ ) that are applied to the reduced mass matrix $\mathbf{M}_{\mathbf{R}}$. Taking advantage from information embedded in the original mass matrix $\mathbf{M}$ (calling $n_{x}, n_{y}$ and $n_{z}$ its translational d.o.f.'s and calling $m n_{x}, m n_{y}$ and $m n_{z}$ translational master d.o.f.'s), $\mathbf{M}_{\mathbf{R}}^{\text {corr }}$ is determined:

$\mathbf{M}=\left[\begin{array}{lll}\mathbf{M}_{\mathbf{x x}} & \mathbf{M}_{\mathbf{y x}} & \mathbf{M}_{\mathbf{z x}} \\ \mathbf{M}_{\mathbf{x y}} & \mathbf{M}_{\mathbf{y y}} & \mathbf{M}_{\mathbf{z y}} \\ \mathbf{M}_{\mathbf{x z}} & \mathbf{M}_{\mathbf{y z}} & \mathbf{M}_{\mathbf{z z}}\end{array}\right] ;$

$M_{R}=\left[\begin{array}{lll}M_{R x x} & M_{R y x} & M_{R z x} \\ M_{R x y} & M_{R y y} & M_{R z y} \\ M_{R x z} & M_{R y z} & M_{R z z}\end{array}\right] ;$

$\alpha_{\mathrm{M}}=\frac{\sum_{i=1}^{n_{x}} \sum_{j=1}^{n_{x}} M_{x x i, j}}{\sum_{i=1}^{m n_{x}} \sum_{j=1}^{m n_{x}} M_{R_{x x} i, j}} ; \quad \beta_{\mathrm{M}}=\frac{\sum_{i=1}^{n_{y}} \sum_{j=1}^{n_{y}} M_{y y i, j}}{\sum_{i=1}^{m n_{y}} \sum_{j=1}^{m n_{y}} M_{R y y i, j}} ;$

$\gamma_{\mathrm{M}}=\frac{\sum_{i=1}^{n_{z}} \sum_{j=1}^{n_{z}} M_{z z i, j}}{\sum_{i=1}^{m n_{z}} \sum_{j=1}^{m n_{z}} M_{R z z i, j}}$

$$
\left\{\begin{array}{l}
\mathbf{M}_{\mathbf{R x x}}^{\text {corr }}=\alpha_{\mathbf{M}} \cdot \mathbf{M}_{\mathbf{R x x}} \\
\mathbf{M}_{\mathbf{R y y}}^{\text {corr }}=\beta_{\mathbf{M}} \cdot \mathbf{M}_{\mathbf{R y y}} \\
\mathbf{M}_{\mathbf{R z z}}^{\text {corr }}=\gamma_{\mathbf{M}} \cdot \mathbf{M}_{\mathbf{R z z}}
\end{array}\right.
$$

The cross terms (e.g. $\mathbf{M}_{\mathbf{x y}}$ ) are all null if the mass property is isotropic; this is a generally obvious condition unless non isotropic masses are considered.

\subsection{Use of distributed stiffness matrices to condense mass matrix}

The stiffness matrix $\mathbf{K}$ of a general structure is represented as a semi-positive definite symmetric matrix containing diagonal $\left(k_{l l}\right)$ and off-diagonal terms $\left(k_{i j}\right.$ - that can be equal or different to zero).

In Eq. (40) the sum is performed on both diagonal and offdiagonal terms of the matrix:

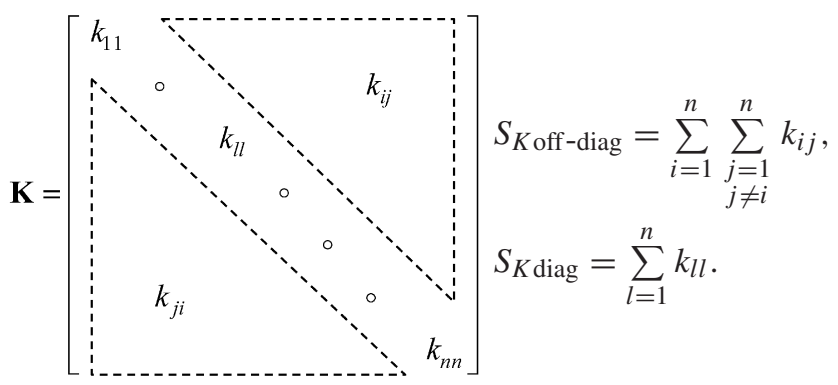

Examining previous Eq. (15) it clearly results that, whenever most of the off-diagonal terms vanish, i.e. $\mathbf{K}_{\mathbf{m s}}^{(\mathbf{G})}=\mathbf{K}_{\mathbf{s m}}^{(\mathbf{G})} \bumpeq 0$, the resulting condensed mass matrix $\mathbf{M}_{\mathbf{R}}$ approaches $\mathbf{M}_{\mathbf{m m}}$.

Among many possible ways to introduce more connected stiffness matrices (less zeros terms), an efficient approach is here proposed. The aim is to balance diagonal and off-diagonal subsets. Taking advantage of the normalisation that makes use of variables introduced in Eq. (40), two additional scalars $\delta$ and $\eta$ are introduced in order to parameterize the elements in the off-diagonal matrix subset.

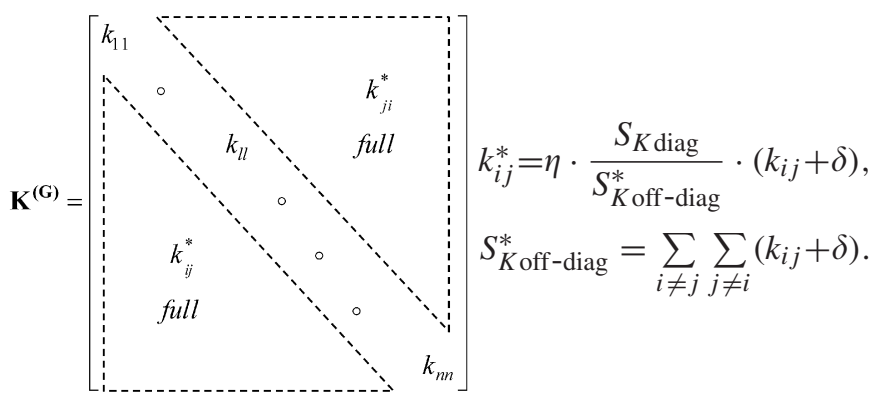

A series of numerical tests demonstrated that the resulting condensed mass matrix is affected by $\mathbf{K}^{(\mathbf{G})}$ active connections (non zero values) more than by individual magnitudes. As a matter of fact, a simple $\mathbf{K}_{\mathrm{TEST}}^{(\mathbf{G})}$ can be used obtaining a condensed mass matrix resulting very close to that obtained using previous $\mathbf{K}^{(\mathbf{G})}$ :

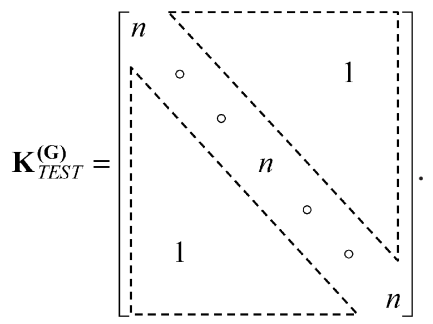


a

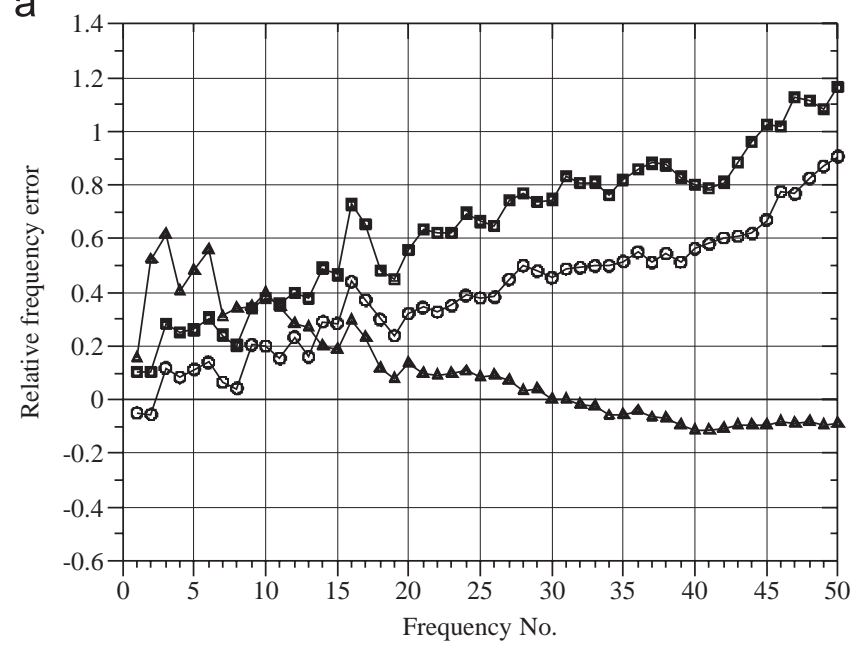

\begin{tabular}{|c|c|c|c|c|c|}
\hline $\begin{array}{c}\text { Freq. } \\
{[\mathrm{SI}]}\end{array}$ & $\begin{array}{c}\text { No } \\
\text { condensed }\end{array}$ & $\begin{array}{l}\text { Classic } \\
\text { Guyan }\end{array}$ & $\begin{array}{c}\text { Guyan } \\
\text { corrected }\end{array}$ & $\begin{array}{c}\text { New } \\
\text { method }\end{array}$ & \\
\hline $1^{\mathrm{st}}$ & 581.3 & 641.2 & 552.6 & 670.5 & \\
\hline $2^{\text {nd }}$ & 602.7 & 665.0 & 569.6 & 917.6 & \\
\hline $3^{\text {rd }}$ & 925.6 & 1185.8 & 1034.5 & 1497.5 & \\
\hline $4^{\text {th }}$ & 1168.1 & 1463.2 & 1265.1 & 1638.7 & \\
\hline $5^{\text {th }}$ & 1230.4 & 1551.7 & 1368.4 & 1823.3 & \\
\hline $10^{\mathrm{th}}$ & 7042.4 & 15241.6 & 13428.3 & 6424.9 & \\
\hline $1^{\mathrm{st}}$ & 1323.1 & 1353.4 & 1131.6 & 2007.4 & \\
\hline $2^{\text {nd }}$ & 2002.9 & 2105.7 & 1756.2 & 2234.9 & \\
\hline $3^{\text {rd }}$ & 2724.3 & 3141.3 & 2493.6 & 3790.9 & \\
\hline $4^{\text {th }}$ & 3703.1 & 3889.5 & 2647.6 & 3958.5 & \\
\hline $5^{\text {th }}$ & 4222.9 & 4695.4 & 3516.1 & 6177.1 & \\
\hline $10^{\text {th }}$ & 38766.4 & 85786.7 & 65378.8 & 33675.3 & \\
\hline $1^{\mathrm{st}}$ & 169.1 & 170.4 & 135.4 & 155.7 & \\
\hline $2^{\text {nd }}$ & 336.3 & 344.3 & 312.5 & 348.7 & \\
\hline $3^{\text {rd }}$ & 483.4 & $\begin{array}{l}496.7 \\
\end{array}$ & $\begin{array}{l}443.8 \\
\end{array}$ & 435.7 & 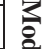 \\
\hline $4^{\text {th }}$ & 927.7 & 1154.1 & 680.3 & 667.3 & \\
\hline $5^{\text {th }}$ & 1104.3 & 1335.1 & 841.7 & 724.0 & \\
\hline $10^{\text {th }}$ & 5721.1 & 10446.9 & 9337.5 & 4633.5 & \\
\hline
\end{tabular}

b

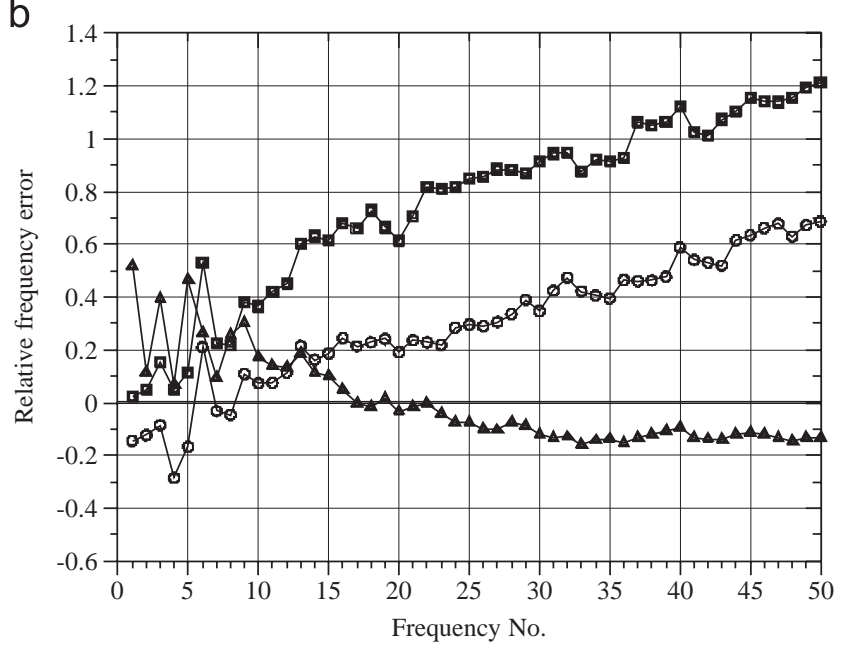

C

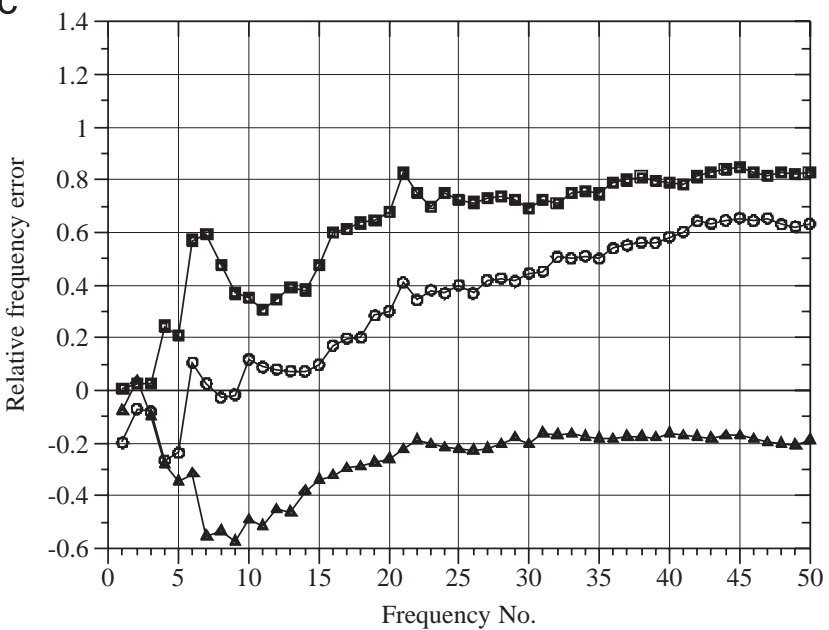

\begin{tabular}{|l|l|l|}
\cline { 2 - 3 } \multicolumn{1}{c|}{} & Guyan corrected & New method \\
\hline $\mathrm{nm}_{\mathrm{x}}=13$ & $\alpha_{\mathrm{M}}=\mathbf{1 . 7 3}$ & $\alpha_{\mathrm{M}}=\mathbf{2 3 . 8 9}$ \\
\hline $\mathrm{nm}_{\mathrm{y}}=24$ & $\beta_{\mathrm{M}}=\mathbf{1 . 3 9}$ & $\beta_{\mathrm{M}}=\mathbf{1 8 . 6 0}$ \\
\hline $\mathrm{nm}_{\mathrm{z}}=38$ & $\gamma_{\mathrm{M}}=\mathbf{1 . 6 9}$ & $\gamma_{\mathrm{M}}=\mathbf{5 . 3 3}$ \\
\hline
\end{tabular}

\begin{tabular}{|l|l|l|}
\cline { 2 - 3 } \multicolumn{1}{c|}{} & Guyan corrected & New method \\
\hline $\mathrm{nm}_{\mathrm{x}}=38$ & $\alpha_{\mathrm{M}}=\mathbf{1 . 1 1}$ & $\alpha_{\mathrm{M}}=\mathbf{4 . 8 9}$ \\
\hline $\mathrm{nm}_{\mathrm{y}}=37$ & $\beta_{\mathrm{M}}=\mathbf{1 . 1 1}$ & $\beta_{\mathrm{M}}=\mathbf{4 . 9 9}$ \\
\hline $\mathrm{nm}_{\mathrm{z}}=10$ & $\gamma_{\mathrm{M}}=\mathbf{4 . 3 9}$ & $\gamma_{\mathrm{M}}=\mathbf{6 0 . 6 9}$ \\
\hline
\end{tabular}

Fig. 5. Comparison of eigenfrequency predictions among original Guyan method ( $\mathbf{E}-$ ), mass corrected Guyan method ( $\longrightarrow$ ○ method (- $\mathbf{\Delta}$ ), using as many master d.o.f.'s as $\frac{1}{8}$ of total number d.o.f.'s, for the three models considered: (a) Model 1; (b) Model 2; and (c) Model 3.

The procedures just described are schematically synthesized in Fig. 1 that shows both the use of the mass correction and the application of the modified $\mathbf{K}^{(\mathbf{G})}$.

\section{Applications and method efficiency}

The following examples are referred to three models discretized by means of brick eight-nodes finite elements, Fig. 2 . Model 1 represents a quarter of a spherical dome, that is a symmetric structure with few inter-element connections; the model presents many repeated eigenfrequencies. Model 2 has similar inter-element connections, but no symmetry is encountered. Model 3 differs from all others because it is very massive, with several connections among elements.

In the first case examined the condensation is very intensive, presenting only ten master nodes. Fig. 3 shows the effect of mass compensation applied to Guyan reduction-computed with Eqs. (38) and (39)—on eigenfrequency evaluation errors. 
a

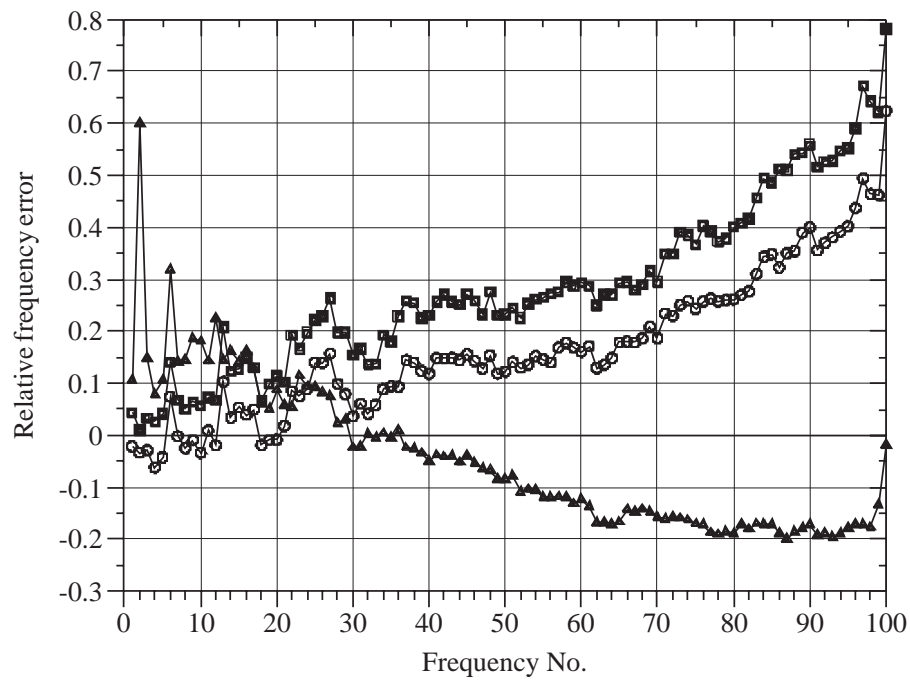

\begin{tabular}{|l|l|l|}
\cline { 2 - 3 } \multicolumn{1}{c|}{} & Guyan corrected & New method \\
\hline $\mathrm{nm}_{\mathrm{x}}=38$ & $\alpha_{\mathrm{M}}=\mathbf{1 . 1 6}$ & $\alpha_{\mathrm{M}}=\mathbf{4 . 1 2}$ \\
\hline $\mathrm{nm}_{\mathrm{y}}=31$ & $\beta_{\mathrm{M}}=\mathbf{1 . 1 6}$ & $\beta_{\mathrm{M}}=\mathbf{4 . 7 6}$ \\
\hline $\mathrm{nm}_{\mathrm{z}}=31$ & $\gamma_{\mathrm{M}}=\mathbf{1 . 1 6}$ & $\gamma_{\mathrm{M}}=\mathbf{4 . 7 6}$ \\
\hline
\end{tabular}

b

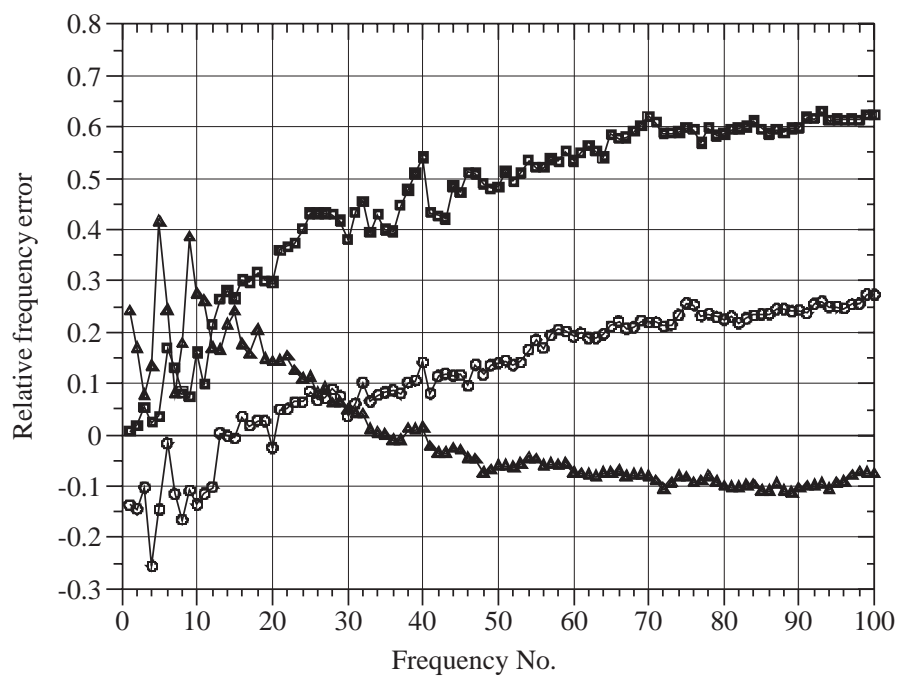

\begin{tabular}{|l|l|c|}
\cline { 2 - 3 } \multicolumn{1}{c|}{} & Guyan corrected & New method \\
\hline $\mathrm{nm}_{\mathrm{x}}=25$ & $\alpha_{\mathrm{M}}=\mathbf{1 . 7 2}$ & $\alpha_{\mathrm{M}}=\mathbf{1 1 . 1 8}$ \\
\hline $\mathrm{nm}_{\mathrm{y}}=67$ & $\beta_{\mathrm{M}}=\mathbf{1 . 2 9}$ & $\beta_{\mathrm{M}}=\mathbf{4 . 1 5}$ \\
\hline $\mathrm{nm}_{\mathrm{z}}=58$ & $\gamma_{\mathrm{M}}=\mathbf{1 . 6 5}$ & $\gamma_{\mathrm{M}}=\mathbf{3 . 2 6}$ \\
\hline
\end{tabular}

\begin{tabular}{|c|c|c|c|c|}
\hline $\begin{array}{l}\text { Freq. } \\
{[\mathrm{SI}] .}\end{array}$ & $\begin{array}{c}\text { No } \\
\text { condensed }\end{array}$ & $\begin{array}{l}\text { Classic } \\
\text { Guyan }\end{array}$ & $\begin{array}{c}\text { Guyan } \\
\text { corrected }\end{array}$ & $\begin{array}{c}\text { New } \\
\text { method }\end{array}$ \\
\hline $1^{\mathrm{st}}$ & 581.3 & 606.5 & 568.8 & 642.5 \\
\hline $2^{\text {nd }}$ & 602.7 & $\begin{array}{l}608.8 \\
\end{array}$ & 5 & 963.7 \\
\hline $3^{\mathrm{rd}}$ & 925.6 & 955.3 & 899.0 & 1063.2 \\
\hline $4^{\text {th }}$ & 1168.1 & 1198.0 & 1096.0 & 1260.4 \\
\hline $5^{\text {th }}$ & 1230.4 & 1281.8 & 1178.0 & 1362.0 \\
\hline $50^{\mathrm{th}}$ & 7042.4 & 8683.2 & 7902.9 & 6452.8 \\
\hline $1^{\mathrm{st}}$ & 1323.1 & 1332.9 & 1142.5 & 1641.3 \\
\hline $2^{\text {nd }}$ & 2002.9 & 2040.9 & 1713.7 & 2337.0 \\
\hline $3^{\text {rd }}$ & 2724.3 & 2870.4 & 2445.5 & 2929.6 \\
\hline $4^{\text {th }}$ & 3703.1 & 3795.2 & 2756.3 & 4196.4 \\
\hline $5^{\text {th }}$ & 4222.9 & 4377.1 & 3608.2 & 5971.6 \\
\hline $50^{\text {th }}$ & 38766.4 & 57506.2 & 44205.4 & 36406.5 \\
\hline $1^{\mathrm{st}}$ & 169.1 & 169.4 & 155.8 & 172.0 \\
\hline $2^{\text {nd }}$ & 336.3 & 340.9 & 328.2 & 375.1 \\
\hline $3^{\text {rd }}$ & 483.4 & 489.2 & 463.5 & 492.5 \\
\hline $4^{\text {th }}$ & 927.7 & 992.5 & 860.6 & 850.4 \\
\hline $5^{\text {th }}$ & 1104.3 & 1197.8 & 1109.0 & 1118.5 \\
\hline $50^{\text {th }}$ & 5721.1 & 7713.4 & 7147.1 & 5051.2 \\
\hline
\end{tabular}

C

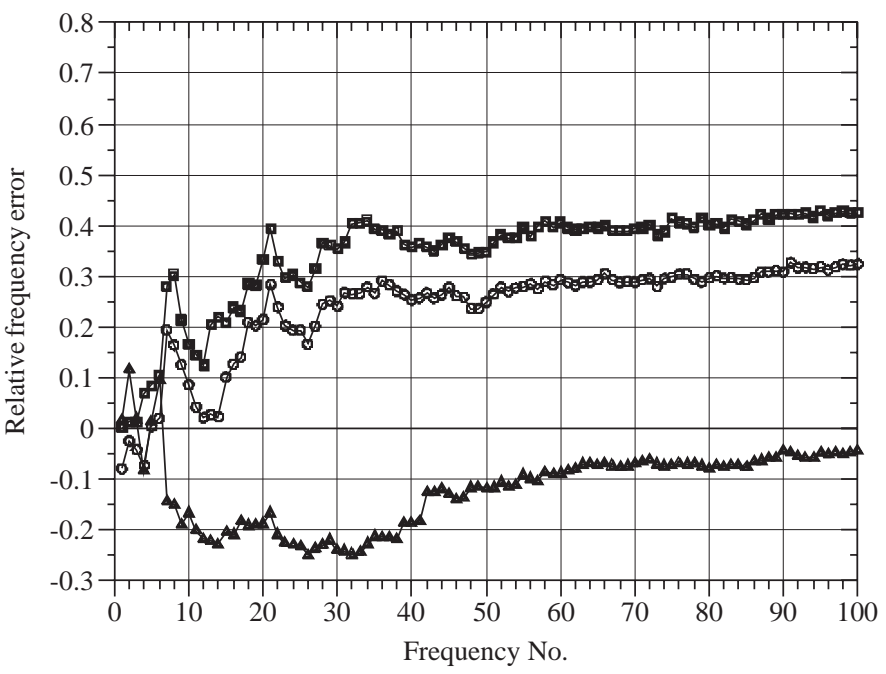

\begin{tabular}{|l|l|c|}
\cline { 2 - 3 } \multicolumn{1}{c|}{} & Guyan corrected & New method \\
\hline $\mathrm{nm}_{\mathrm{x}}=75$ & $\alpha_{\mathrm{M}}=\mathbf{1 . 1 7}$ & $\alpha_{\mathrm{M}}=\mathbf{2 . 2 4}$ \\
\hline $\mathrm{nm}_{\mathrm{y}}=75$ & $\beta_{\mathrm{M}}=\mathbf{1 . 0 8}$ & $\beta_{\mathrm{M}}=\mathbf{2 . 2 4}$ \\
\hline $\mathrm{nm}_{\mathrm{z}}=20$ & $\gamma_{\mathrm{M}}=\mathbf{1 . 3 8}$ & $\gamma_{\mathrm{M}}=\mathbf{1 2 . 5 1}$ \\
\hline
\end{tabular}

Fig. 6. Comparison of eigenfrequency predictions among original Guyan method ( $-\mathbf{E}-$ ), mass corrected Guyan method ( $\longrightarrow \bigcirc-$ - and new developed method (_ $)$, using as many master d.o.f.'s as $\frac{1}{4}$ of total number d.o.f.'s, for the three models considered: (a) Model 1; (b) Model 2; and (c) Model 3.

It is highlighted by graphics, and corresponding tables, that mass correction provides a very important improvement in Guyan method efficiency. In other words, the new mass matrix reduction is now in accordance with the global translational inertia properties of the original model. Furthermore, it is interesting to highlight the amount of mass correction; on purpose, parameters $\alpha_{\mathrm{M}}, \beta_{\mathrm{M}}, \gamma_{\mathrm{M}}$ of Eqs. (38b) are displayed in Figs. 3-6. Considering also the new method that makes use of the $\mathbf{K}^{(\mathbf{G})}$ matrix given in Eq. (41), results turns out to be astonishingly accurate in terms of eigenfrequency identifications. The new mass matrix reduction not only reduces the eigenfrequency estimate error, but also eliminates the 
trend in rising the error while increasing the mode order. This latter tendency is typical in Guyan reduction and cannot be eliminated introducing only mass compensation on Guyan method.

The application of $\mathbf{K}^{(\mathbf{G})}$, defined in Eq. (41), may produce different reduction methods simply by varying parameters $\delta$ and $\eta$. In particular, Fig. 4 shows the results concerning model 2 , for various solution strategies, all including mass correction. The third sequence of data regards a $\mathbf{K}^{(\mathbf{G})}$ built only with its diagonal values. Fourth and fifth sequences are obtained filling up the off-diagonal parts and balancing its weight with respect to matrix diagonal. The sixth sequence is performed using the stiffness matrix given in Eq. (42).

Whatever is the strategy (among the new ones) the results are fine and similar each other. The only constraint is that the $\mathbf{K}^{(\mathbf{G})}$ stiffness has to be semi positive defined. This confirms that it is not essential to use a physical stiffness matrix to build a reduced mass matrix. On the contrary, defining $\mathbf{K}^{(\mathbf{G})}$ stiffness properties by means of a homogeneous criterion improves the results.

Reconsidering Eq. (15), it is clear that the use of $\mathbf{K}^{(\mathbf{G})}$ with $\eta=0$ (Eq. (41)) implies only the omission of all terms after the first in the r.h.s. of Eq. (15); therefore, mass reduction can be obtained using $\mathbf{M}_{\mathbf{m m}}$ with mass correction.

In Figs. 5 and 6 the Guyan reduction with and without mass correction is faced with the new method obtained by Eq. (41) with $\eta=1$; in the figures the number of master d.o.f.'s is amplified to an eighth and a quarter of the total number of d.o.f.'s, respectively. The method shows to be particularly reliable at the higher modes, i.e. after almost the tenth mode the accuracy turns into much better results if compared with Guyan approach. This is particularly emphasized for the first two models. For the third one the results given by application of Eq. (41) present a less marked oscillation on the first modes considered. However, the error trend given by Guyan approach (mass corrected or none), that is almost increasing linearly, is avoided by the new method. Above remarks suggest an optimal strategy for the dynamic mass reduction of structures.

Mass correction, given by Eqs. (39), always provides important benefits to precision. In addition, if condensation is very intensive, the new method is commonly the most reliable. If condensation is low (number of master d.o.f.'s almost equal to total d.o.f.'s) the results suggest a possible mixed use of Guyan compensated approach for lower modes and the new proposed method for the others.
Considering mode shapes, differences are less evident among the considered approaches, so that the matching of mode shapes keeps the same well known difficulties: as everybody knows, the matching results unpredictable at the higher frequencies.

\section{Conclusions}

The mass matrix reduction based on Guyan theory has been shown not to maintain the mass properties of the structure at their known values when restrained structures are faced. Enforcing mass conservation increases the reliability of identified eigenfrequencies. It was also highlighted that there is no need to build the mass reduced matrix by exploiting the effective stiffness matrix of the model. On the contrary, the use of more homogeneous stiffness matrices provides much better results: new opportunities arise to solve large dynamic problems with compact numerical matrices, while maintaining a high accuracy in dynamic analyses.

\section{References}

[1] W.C. Hurty, Dynamic analysis of structural systems using component modes, Am. Inst. Aeronaut. Astronaut. J. 3 (4) (1965) 678-685.

[2] R.R. Craig, M.C.C. Bampton, Coupling of substructures for dynamic analysis, Am. Inst. Aeronaut. Astronaut. J. 6 (7) (1968) 1313-1319.

[3] R.J. Guyan, Reduction of stiffness and mass matrices, Am. Inst. Aeronaut. Astronaut. J. 3 (2) (1965) 380.

[4] R.M. Lin, M.K. Lim, Complex eigensensitivity-based characterization of structures with viscoelastic damping, J. Acoustic Soc. Am. 100 (5) (1996) 3182-3191.

[5] N. Bouhaddi, R. Fillod, A method for selecting master DOF in dynamic substructuring using the Guyan condensation method, Comput. Struct. 45 (5) (1992) 941-946.

[6] J.E.T. Penny, M.I. Friswell, S.D. Garvey, Automatic choice of measurement locations for dynamic testing, Am. Inst. Aeronaut. Astronaut. J. 32 (2) (1994) 407-414.

[7] A.Y.T. Leung, An accurate method of dynamic substructuring with simplified computation, Int. J. Numer. Methods Eng. 14 (1979) $1241-1256$.

[8] J. O'Callahan, A procedure for an improved reduced system (IRS) model, Proceedings of the 7th International Modal Analysis Conference, Las Vegas, 1989, pp. 17-21.

[9] M.I. Friswell, S.D. Garvey, J.E.T. Penny, Model reduction using dynamic and iterated IRS techniques, J. Sound Vib. 186 (2) (1995) 311-323.

[10] M.I. Friswell, S.D. Garvey, J.E.T. Penny, The convergence of the iterated IRS method, J. Sound Vib. 211 (1) (1995) 123-132.

[11] K.-O. Kim, M.-K. Kang, Convergence acceleration of iterative modal reduction methods, Am. Inst. Aeronaut. Astronaut. J. 39 (1) (2001) 134-140.

[12] K.D. Henshell, J.H. Ong, Automatic masters for eigenvalue economization, Earthquake Eng. Struct. Dyn. 3 (1975) 375-383. 